\title{
Exploring the oxygenase function of Form II Rubisco for production of glycolate from $\mathrm{CO}_{2}$
}

Fan Yang ${ }^{1,2,3}$, Junli Zhang ${ }^{1,2,3}$, Zhen Cai ${ }^{1}$, Jie Zhou ${ }^{1,3^{*}}$ and Yin Li $i^{*}$

\begin{abstract}
The oxygenase activity of Ribulose-1,5-bisphosphate carboxylase/oxygenase (Rubisco) converts ribulose-1,5-bisphosphate (RuBP) into 2-phosphoglycolate, which in turn channels into photorespiration, resulting in carbon and energy loss in higher plants. We observed that glycolate can be accumulated extracellularly when two genes encoding the glycolate dehydrogenase of cyanobacteria Synechocystis sp. PCC 6803 were inactivated. This inspired us to explore the oxygenase function of Rubisco for production of glycolate, an important industrial chemical, from $\mathrm{CO}_{2}$ by engineered cyanobacteria. Since the oxygenase activity of Rubisco is generally low in $\mathrm{CO}_{2}$-rich carboxysome of cyanobacteria, we introduced Form II Rubisco, which cannot be assembled in carboxysome, into the cytoplasm of cyanobacteria. Heterologous expression of a Form II Rubisco from endosymbiont of tubeworm Riftia pachyptila (RPE Rubisco) significantly increased glycolate production. We show that the RPE Rubisco is expressed in the cytoplasm. Glycolate production increased upon addition of $\mathrm{NaHCO}_{3}$ but decreased upon supplying $\mathrm{CO}_{2}$. The titer of glycolate reached $2.8 \mathrm{~g} / \mathrm{L}$ in 18 days, a 14 -fold increase compared with the initial strain with glycolate dehydrogenase inactivated. This is also the highest glycolate titer biotechnologically produced from $\mathrm{CO}_{2}$ ever reported. Photosynthetic production of glycolate demonstrated the oxygenase activity of Form II Rubisco can be explored for production of chemicals from $\mathrm{CO}_{2}$.
\end{abstract}

Keywords: Rubisco, Oxygenase activity, Glycolate production, Cyanobacteria, $\mathrm{CO}_{2}$

\section{Introduction}

Ribulose-1,5-bisphosphate

carboxylase/oxygenase (Rubisco) is the key enzyme in photosynthesis (Jensen 2000, Erb et al. 2018). It is responsible for the primary carbon fixation in Calvin-Benson-Bassham (CBB) cycle, catalyzing the addition of $\mathrm{CO}_{2}$ to ribulose-1,5-bisphosphate (RuBP), leading to the formation of 3-phosphoglycerate (3PGA) (Moroney et al. 2013). Despite its pivotal role in the biosphere, Rubisco is notorious for its poor carboxylation activity and specificity (Davidi et al. 2020). The poor specificity of Rubisco is due to its oxygenase activity, as $\mathrm{CO}_{2}$ and $\mathrm{O}_{2}$ are competitive substrates of

\footnotetext{
*Correspondence: jiezhouw@im.ac.cn; yli@im.ac.cn

${ }^{1}$ CAS Key Laboratory of Microbial Physiological and Metabolic Engineering, State Key Laboratory of Microbial Resources, Institute of Microbiology, Chinese Academy of Sciences, Beijing 100101, China

${ }^{3}$ CAS Key Laboratory of Microbial Physiological and Metabolic Engineering, State Key Laboratory of Transducer Technology, Institute of Microbiology, Chinese Academy of Sciences, Beijing 100101, China Full list of author information is available at the end of the article
}

Rubisco (Moroney et al. 2013). The oxygenation reaction catalyzed by the oxygenase activity of Rubisco results in the production of 2-phosphoglycolate (2PG) (Eisenhut et al. 2008a, b). Although 2PG can be metabolized through photorespiration and recycled back into the central carbon metabolism, this process is energy-consuming and leads to carbon loss (Moroney et al. 2013, Fernie et al. 2020).

The oxygenase activity of Rubisco is often considered undesirable but unavoidable (Moroney et al. 2013). A compelling evidence is that active photorespiration is found in all oxygenic photosynthetic organisms to metabolize 2PG, the toxic oxygenation product of Rubisco (Moroney et al. 2013). Engineering Rubisco for an improved carboxylation efficiency often comes at a price of decreased $\mathrm{CO}_{2}: \mathrm{O}_{2}$ specificity, not to mention the complete removal of its oxygenase activity (Davidi et al. 2020). In fact, there are no $\mathrm{CO}_{2}$ or $\mathrm{O}_{2}$ binding sites found in Rubisco (Moroney et al.2013). Rubisco binds RuBP and 
converts it to the 2,3-enediol form, allowing the subsequent addition of either $\mathrm{CO}_{2}$ or $\mathrm{O}_{2}$ (Spreitzer et al. 2002). Due to this catalytic mechanism of Rubisco, it is proposed that the oxygenation reaction of Rubisco cannot be eliminated by mutation (Moroney et al. 2013).

Since the oxygenation function of Rubisco cannot be avoided, and the oxygenation product is involved in the overall carbon metabolism, we propose we can take this advantage to employ the oxygenase activity of Rubisco to produce useful chemicals. In Synechocystis sp. PCC 6803 (hereafter Synechocystis), 2PG is subsequently converted to glycolate, an important $\alpha$-hydroxy acid with a wide range of industrial applications in cosmetics, pharmaceuticals and biodegradable polymeric material production (Eisenhut et al. 2006, 2008a, b; Zahoor et al. 2014; Zhan et al. 2020). Especially, the polymer of glycolate (PGA) and the co-polymer of glycolate and lactate (PGLA) are both excellent biodegradable materials with medical applications (Salusjärvi et al. 2017). In engineered heterotrophic cell factories including Escherichia coli and Saccharomyces cerevisiae, glycolate could be produced from glucose through the glyoxylate shunt or from xylose through xylulose-1-phosohate pathway and xylose oxidation pathway (Koivistoinen et al. 2013; Deng et al. 2015; Alkim et al. 2016; Liu et al. 2018). Recently, a novel glycolate synthetic pathway from glycerol was also successfully constructed in E. coli (Zhan et al. 2020). To date, the highest titer of $65.5 \mathrm{~g} / \mathrm{L}$ with a yield of $0.79 \mathrm{~g} / \mathrm{g}$ glucose was obtained by balancing the flux distribution between the TCA cycle and glyoxylate shunt in E. coli (Deng et al. 2018). However, the production of glycolate from $\mathrm{CO}_{2}$ in cyanobacteria has never been reported.

Thus, we intended to produce glycolate from $\mathrm{CO}_{2}$ using the oxygenase activity of Rubisco in Synechocystis, providing a unique application avenue of the oxygenase activity in photosynthetic biosynthesis.

\section{Methods and material}

\section{Plasmids and strains construction}

All plasmids constructed in this study were summarized in Additional file 1: Table S1. Escherichia coli DH5 $\alpha$ was used as the host for plasmids construction. All plasmids were generated through Gibson Assembly (NEB, China) of amplified inserts and linearized pUC57 plasmid backbones with primers designed using NEBuilder Assembly Tool (http://nebuilder.neb.com/). All Synechocystis mutant strains constructed in this study were summarized in Additional file 1: Table S1. Cyanobacterial strains were generated by transforming cells with certain plasmids which included homologous regions as well as the inserts. Rubiscos were individually overexpressed under the control of the promoter $\mathrm{P}_{\text {cpc560 }}$. The DNA cassette together with a chloromycetin resistance marker was integrated into the pta site (slr2132) of Synechocystis genome. Transformation of Synechocystis was performed as previously described (Lindberg et al. 2010). The colonies were selected on BG-11 plates supplemented with single or combined antibiotics $(10 \mu \mathrm{g} / \mathrm{mL}$ chloromycetin, $30 \mu \mathrm{g} / \mathrm{mL}$ erythromycin, $10 \mu \mathrm{g} / \mathrm{mL}$ spectinomycin). Complete segregation and correct gene insertions were checked by PCR and sequencing with primers listed in Additional file 1: Table S2.

\section{Culture conditions}

All strains were grown in $50 \mathrm{~mL}$ erlenmeyer flask containing $20 \mathrm{~mL}$ of BG11 medium at $30^{\circ} \mathrm{C}$ under a constant illumination intensity of $100 \mu \mathrm{mol}$ photons $\mathrm{m}^{-2} \mathrm{~s}^{-1}$, with atmospheric $\mathrm{CO}_{2}$ level or supplemented with prescribed concentration of $\mathrm{NaHCO}_{3}$. The initial $\mathrm{OD}_{730}$ was normalized to 0.5 . Antibiotics were added to the culture for routine maintenance of mutants when necessary. Growth was monitored by measurement of the optical density at $730 \mathrm{~nm}\left(\mathrm{OD}_{730}\right)$ every three days.

\section{Quantification of extracellular glycolate concentration}

Extracellular glycolate concentration was determined using the culture supernatant every three days. $10 \mu \mathrm{L}$ of culture supernatant was analyzed by HPLC equipped with Bio-Rad Aminex ${ }^{\circledR}$ HPX-87H Ion Exclusion Column $(300 \mathrm{~mm} \times 7.8 \mathrm{~mm})$ using $8 \mathrm{mM} \mathrm{H}_{2} \mathrm{SO}_{4}$ as mobile phase, pumped at a flow rate of $0.6 \mathrm{~mL} / \mathrm{min}$. The column temperature was maintained at $50^{\circ} \mathrm{C}$, and peaks were detected using Agilent Technologies 1260 RID (refractive index detector).

\section{Quantification of intracellular 2PG and glycolate concentration}

The intracellular concentrations of 2PG and glycolate were determined after three days of cultivation. To rapidly quench the cell metabolism, $5 \mathrm{~mL}$ of cultures were cooled to $0{ }^{\circ} \mathrm{C}$ within $15 \mathrm{~s}$ in a $-50{ }^{\circ} \mathrm{C}$ methanol bath. After centrifugation at $4{ }^{\circ} \mathrm{C}$ for $5 \mathrm{~min}$ at $8000 \times g$, the cell pellets were washed once with precooled water and resuspended in $2 \mathrm{~mL}$ of precooled $80 \%$ ( $\mathrm{vol} / \mathrm{vol}$ ) methanol solution. After incubation at $20^{\circ} \mathrm{C}$ for $30 \mathrm{~min}$, the samples were then centrifuged at $4{ }^{\circ} \mathrm{C}$ for $10 \mathrm{~min}$ at $20,000 \times g$. The supernatants were dried by lyophilization and redissolved in $200 \mu \mathrm{L}$ of water.

The concentrations of 2PG and glycolate were determined with AB Sciex Qtrap 6500 LC-MS/MS System. Injection volume was $5 \mu \mathrm{L}$. Metabolites were separated with a HyperREZ XP Organic acid column $(100 \times 7.7 \mathrm{~mm}$, Thermo Fisher Scientific $)$ with $\mathrm{H}_{2} \mathrm{O}$ as the solvent. The column was maintained at $40{ }^{\circ} \mathrm{C}$ with a solvent flow rate of $0.4 \mathrm{~mL} / \mathrm{min}$. The electrospray ionization MS was operated in the negative ion mode. The mass 
spectra was acquired in multiple-reaction monitoring model for the optimized ion pairs of 2PG and glycolate.

\section{SDS PAGE and Native PAGE}

To prepare the protein samples for SDS PAGE and Native PAGE, Synechocystis cells were harvested by centrifugation and resuspended with $1 \mathrm{~mL}$ buffer $(50 \mathrm{mM}$ Tris- $\mathrm{HCl}$, $\mathrm{pH} 8.0,10 \mathrm{mM} \mathrm{MgCl}_{2}, 1 \mathrm{mM}$ EDTA) for ultrasonication. After centrifugation, the supernatants were mixed with SDS loading buffer or Native loading buffer at 1:1. The protein samples were detected with SDS PAGE or native PAGE after the total protein amount was normalized to $7 \mu \mathrm{g}$.

\section{Fluorescence microscopy}

$5 \mu \mathrm{L} \log$-phase cells were spotted onto $1 \%(\mathrm{w} / \mathrm{v}$ in BG11) agarose pads and air-dried before application of a $0.17 \mathrm{~mm}$ coverglass. Fluorescence microscopy was performed on a Nikon N-SIM S Super Resolution Microscope with a $63 \mathrm{x} / 1.4 \mathrm{NA}$ oil-immersion objective using laser lines at $488 \mathrm{~nm}$ and $561 \mathrm{~nm}$.

\section{Results}

Inactivation of two genes encoding glycolate dehydrogenase in Synechocystis resulted in glycolate production

In Synechocystis, glycolate is converted to glyoxylate by two glycolate dehydrogenases (GlcD1 and GlcD2), and subsequently metabolized by three branched routes (Eisenhut et al. 2006, Eisenhut M, Ruth W Eisenhut et al. 2008a, b). To completely block the glycolate metabolism, both GlcD1 and GlcD2 encoded by $g l c D 1$ and $g l c D 2$, respectively, were inactivated (Fig. 1). The resulting mutant was designated as WT- $\Delta$ glcD (Table 1). Complete segregation and correct gene insertions at both $g l c D 1$ and $g l c D 2$ sites were verified by PCR and sequencing (Additional file 1: Fig. S1).

As glycolate metabolism was completely blocked, we next investigated glycolate accumulation in strain WT- $\Delta$ glcD. Both the intracellular and extracellular glycolate concentrations of WT- $\Delta$ glcD were analyzed and compared with that of the WT strain. Samples were taken after three days cultivation supplemented with or without $50 \mathrm{mM} \mathrm{NaHCO}_{3}$. The intracellular glycolate concentration of the WT strain was $0.004 \mu \mathrm{mol} \mathrm{L}^{-1} \mathrm{OD}_{730}{ }^{-1}$

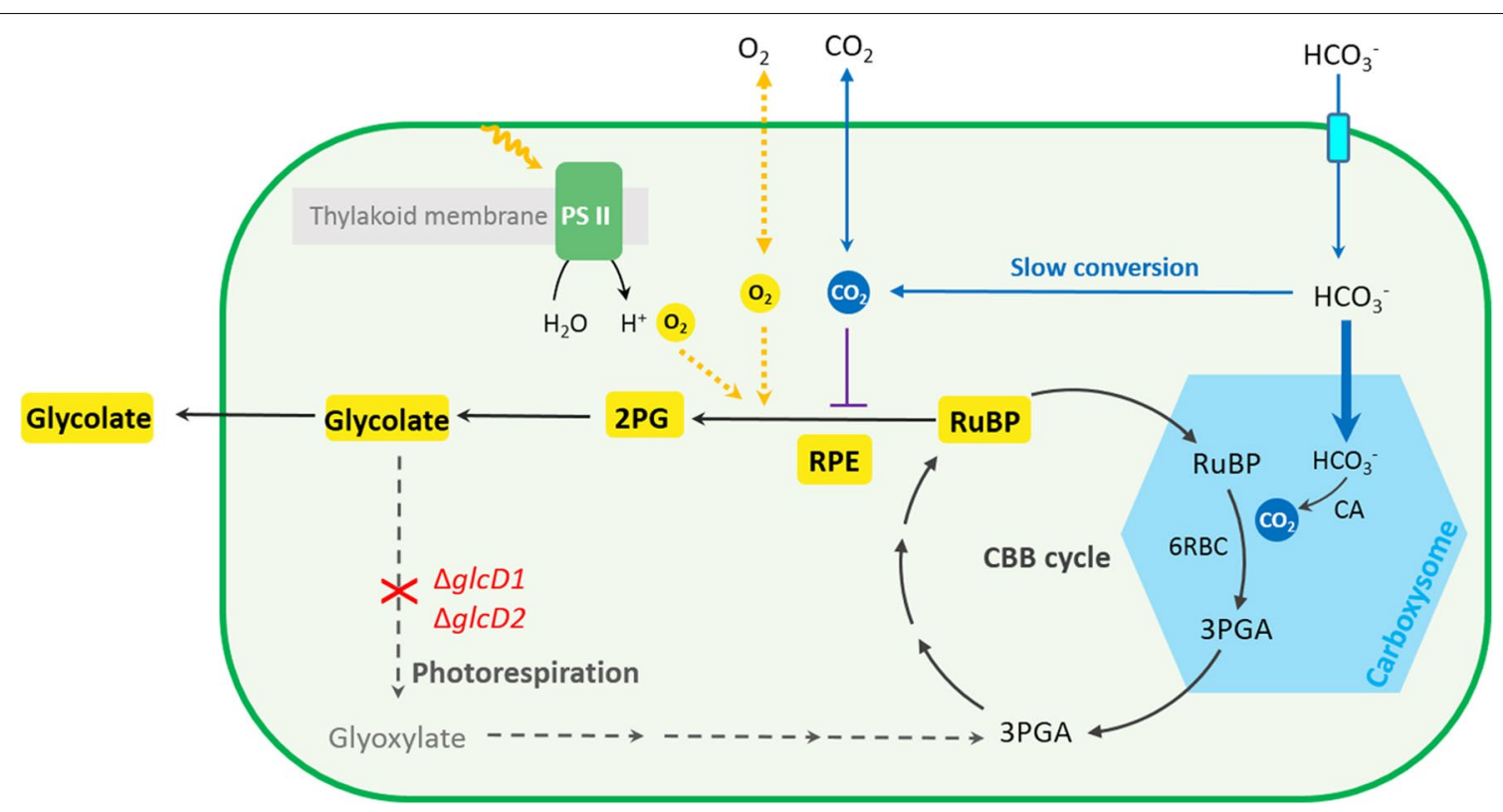

Fig. 1 Diagram of glycolate production via oxygenation function of Form II Rubisco in Synechocystis sp. PCC 6803. $\mathrm{HCO}_{3}{ }^{-}$is actively pumped (light blue) into the cytosol. While some of $\mathrm{HCO}_{3}{ }^{-}$spontaneously converts to $\mathrm{CO}_{2}$ in cytosol, most of $\mathrm{HCO}_{3}{ }^{-}$enters the carboxysome (blue hexagon) and is converted to $\mathrm{CO}_{2}$ by the sequestered carbonic anhydrase (CA). RuBP enters the carboxysome and the sequestered Rubisco of Synechocystis (6RBC Rubisco) combines RuBP with $\mathrm{CO}_{2}$ to generated two molecules of 3-phophoglycerate (3PGA). 3PGA escapes to the cytosol and RuBP is regenerated through the Calvin-Benson-Bassham (CBB) cycle. Photorespiration (gray dashed arrows) can be blocked by inactivating (red cross) two glycolate dehydrogenases (GlcDs) encoded by glcD1 and glcD2, respectively. The resulting strain WT- $\triangle \mathrm{glcD}$ accumulates and excretes glycolate to the culture (yellow). Form II Rubisco from the endosymbiont of Riftia pachyptila (RPE Rubisco) was overexpressed in strain WT- $\triangle \mathrm{glcD}$ and located in the cytosol. As cyanobacteria performs oxygenic photosynthesis, RPE Rubisco catalyzes the oxygenation of RuBP to 2-photoglycolate (2PG), facilitating glycolate production. $\mathrm{As}_{\mathrm{CO}}$ can freely diffuse to the cytosol (blue solid arrow), the elevated $\mathrm{CO}_{2}$ level inhibits the oxygenase function of RPE (purple solid line) and decreases glycolate production when the external inorganic carbon is supplied with $\mathrm{CO}_{2}$ 
Table 1 The Synechocystis strains used in this study

\begin{tabular}{|c|c|c|}
\hline Strain & Genetic background & Source of Rubisco \\
\hline Wild type & Synechocystis sp. PCC 6803 & - \\
\hline WT- $\Delta g l_{C D}$ & WT $\Delta g / c D 1:: \mathrm{em}^{r} ; \Delta g / \mathrm{cD} 2::$ spec $^{r}$ & - \\
\hline$R P E-\triangle g l c D$ & $\Delta g l c D \Delta p t a:: P_{\mathrm{cpc} 560}-r p e^{-T_{\mathrm{rbcs}}}-\mathrm{cm}^{r}$ & Riftia pachyptila endosymbiont \\
\hline $4 \mathrm{Pm}-\triangle \mathrm{glcD}$ & $\Delta g l c D \Delta p t a:: \mathrm{P}_{\mathrm{cpc} 560}-4 p m-\mathrm{T}_{\mathrm{rbcs}}-\mathrm{cm}^{r}$ & Phaeospirillum molischianum \\
\hline $5 S t-\triangle g l c D$ & $\Delta g l c D \Delta p t a:: P_{\mathrm{cpc} 560}-5 s t-\mathrm{T}_{\mathrm{rbcs}}-\mathrm{cm}^{r}$ & Sedimenticola thiotaurini \\
\hline $6 \mathrm{Rbc}-\triangle \mathrm{glcD}$ & $\Delta g l c D \Delta p t a:: P_{c p c 560}-6 r b c L-6 r b c S-T_{r b c s}-c m^{r}$ & Synechocystis sp. PCC 6803 \\
\hline
\end{tabular}

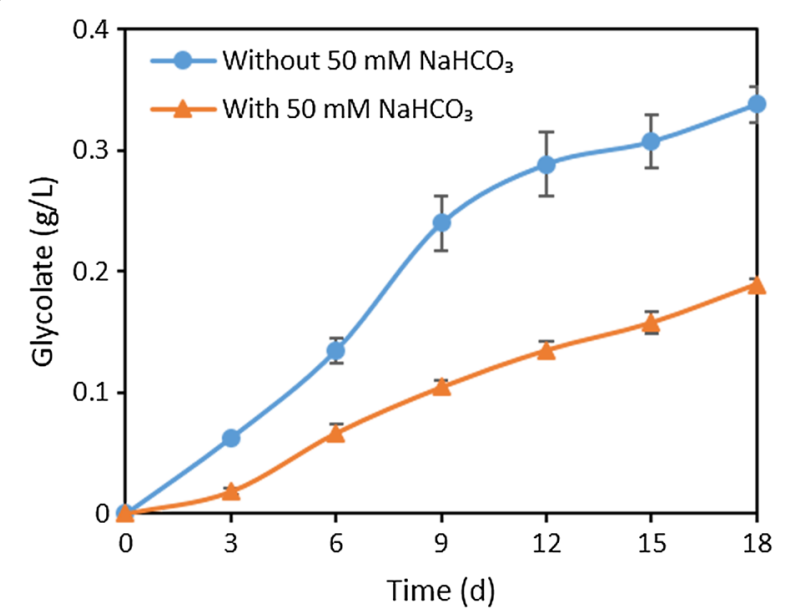

Fig. 2 Glycolate production by strain WT- $\triangle \mathrm{glcD}$ with or without supply of $50 \mathrm{mM} \mathrm{NaHCO}$. The cells were cultivated at $30{ }^{\circ} \mathrm{C}$ under $100 \mu \mathrm{mol}$ photons $\mathrm{m}^{-2} \mathrm{~s}^{-1}$ light intensity. Error bars represent standard deviations from biological triplicates conducted in three independent experiments

and $0.02 \mu \mathrm{mol} \mathrm{L} \mathrm{L}^{-1} \mathrm{OD}_{730}{ }^{-1}$ respectively, when supplemented with or without $50 \mathrm{mM} \mathrm{NaHCO} 3$ (Additional file 1: Fig. S2). Moreover, the extracellular glycolate concentration was undetectable in the WT strain under both conditions (data not shown). It is evident that glycolate could be rapidly metabolized in the WT strain. On the contrary, strain WT- $\Delta$ glcD accumulated glycolate intracellularly and extracellularly under both conditions (Fig. 2 and Additional file 1: Fig. S2). The intracellular glycolate concentration of strain WT- $\Delta$ glcD was $0.51 \mu \mathrm{mol}$ $\mathrm{L}^{-1} \mathrm{OD}_{730}{ }^{-1}$ when supplied with $50 \mathrm{mM} \mathrm{NaHCO}$, and increased to $1.75 \mu \mathrm{mol} \mathrm{L}^{-1} \mathrm{OD}_{730}{ }^{-1}$ without the supply of $\mathrm{NaHCO}_{3}$ (Additional file 1: Fig. S2). Furthermore, the glycolate concentration in the medium of strain WT- $\Delta$ glcD reached $86.47 \mu \mathrm{mol} \mathrm{L} \mathrm{L}^{-1} \mathrm{OD}_{730}{ }^{-1}$ (mass concentration of $0.02 \mathrm{~g} / \mathrm{L}$ ) and $317.77 \mu \mathrm{mol} \mathrm{L}{ }^{-1} \mathrm{OD}_{730}{ }^{-1}$ (mass concentration of $0.06 \mathrm{~g} / \mathrm{L}$ ) after 3 days cultivation respectively, with or without $50 \mathrm{mM} \mathrm{NaHCO}_{3}$ (Fig. 2). Apparently, the majority of glycolate was excreted to the culture by strain WT- $\Delta$ glcD, and the intercellular glycolate accumulation could be negligible. We further monitored the glycolate concentration in the medium every three days and found that strain WT- $\Delta \mathrm{glcD}$ produced $0.19 \mathrm{~g} / \mathrm{L}$ and $0.34 \mathrm{~g} / \mathrm{L}$ of glycolate after 18 days cultivation respectively with or without the supply of $50 \mathrm{mM} \mathrm{NaHCO}$ (Fig. 2). In other words, glycolate can be produced from $\mathrm{CO}_{2}$ and secreted extracellularly upon inactivation of the two glycolate dehydrogenases in Synechocystis. Moreover, strain WT- $\Delta$ glcD produces higher concentration of glycolate when no additional $\mathrm{NaHCO}_{3}$ was supplemented, suggesting ambient level $\mathrm{CO}_{2}$ is sufficient for glycolate production to occur.

\section{Overexpression of the native carboxysome-located Rubisco didn't contribute to glycolate production}

Given the multiple industrial applications of glycolate, we were encouraged to further increase glycolate production. Glycolate synthetic pathway comprises two reactions (Fig. 1). RuBP reacts with $\mathrm{O}_{2}$ to generate one molecule of 2PG and one molecule of 3-phosphoglycerate (3PGA) (Eisenhut et al. 2008a, b, Fernie and Bauwe 2020). 2PG is then dephosphorylated to glycolate and 3PGA enters the CBB cycle to regenerate RuBP (Eisenhut et al. 2008a, b, Fernie and Bauwe 2020). In order to identify the bottleneck of glycolate production, the intercellular 2PG concentrations in the WT strain and strain WT- $\Delta$ glcD were measured. Samples were taken after three days cultivation with or without the supply of $50 \mathrm{mM} \mathrm{NaHCO}$. With the intact glycolate metabolism, the intracellular 2PG concentration in the WT

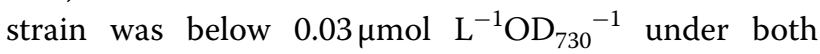
growth conditions (Additional file 1: Fig. S2). The intracellular 2PG level in strain WT- $\Delta$ glcD was at the same level as compared to the WT strain. However, as mentioned above, the intracellular glycolate concentration in strain WT- $\Delta$ glcD became about 100 -fold higher than that of the WT strain irrespective of the supply of $50 \mathrm{mM} \mathrm{NaHCO}$ (Additional file 1: Fig. S2). This indicated that the conversion from 2PG to glycolate in strain WT- $\Delta$ glcD was efficient, which is in line with the discovery that up to four 2-phosphoglycolate phosphatases (PGPase) were identified in Synechocystis to catalyze this 
reaction (Rai et al. 2018). Thus, the oxygenation of RuBP catalyzed by Rubisco was the rate-limiting step of glycolate production.

Thus, to increase glycolate production, the native Rubisco of Synechocystis was overexpressed in strain WT- $\Delta$ glcD. The resulting mutant was designated as strain $6 \mathrm{Rbc}-\Delta \mathrm{glcD}$ (Table 1 ) and its capacity for glycolate production was determined with the same growth conditions as mentioned above. After 18 days of cultivation, strain $6 \mathrm{Rbc}-\Delta \mathrm{glcD}$ produced $0.16 \mathrm{~g} / \mathrm{L}$ and $0.35 \mathrm{~g} / \mathrm{L}$ of glycolate when supplied with or without $50 \mathrm{mM} \mathrm{NaHCO}$, respectively. Neither titer is significantly higher than that of strain WT- $\Delta$ glcD under the same condition (Fig. 3a and $b$ ). In addition, no significant difference was observed in the growth rates of strains $6 \mathrm{Rbc}-\Delta \mathrm{glcD}$ and WT- $\Delta \mathrm{glcD}$ under both conditions (Fig. $3 \mathrm{c}$ and $\mathrm{d}$ ). Moreover, the SDS PAGE and native PAGE results suggested that 6 RBC was successfully overexpressed and assembled under both conditions (Additional file 1: Fig. S4). These results together suggested that overexpression of 6RBC Rubisco did not contribute to increase glycolate production. The reason behind is likely that the native 6RBC Rubisco is encapsulated in a microcompartment found in all cyanobacteria, termed as the carboxysome. It reduces the oxygenase activity of Rubisco by inhibiting the entrance of $\mathrm{O}_{2}$ and increasing $\mathrm{CO}_{2}$ concentration around Rubisco (Espie et al. 2011). Thus, to increase glycolate production, the selected Rubisco is expected to be located outside the carboxysome so as its oxygenase activity can play a role.

\section{Overexpression of Form II rubiscos enhanced glycolate production}

It was previously reported that replacing the native Rubisco of cyanobacteria with Form II Rubisco could not support the biogenesis of carboxysome, indicating Form II Rubisco resides outside the carboxysome (Baker et al. 1998; Durao et al. 2015). If the Rubisco is located in the cytosol, it is accessible to molecule oxygen and a reduced $\mathrm{CO}_{2}$ level due to the absence of carbonic anhydrase in the cytosol (Price et al. 2008; Price 2011). Thus, we hypothesized that Form II Rubiscos might be promising candidates to increase glycolate production. To
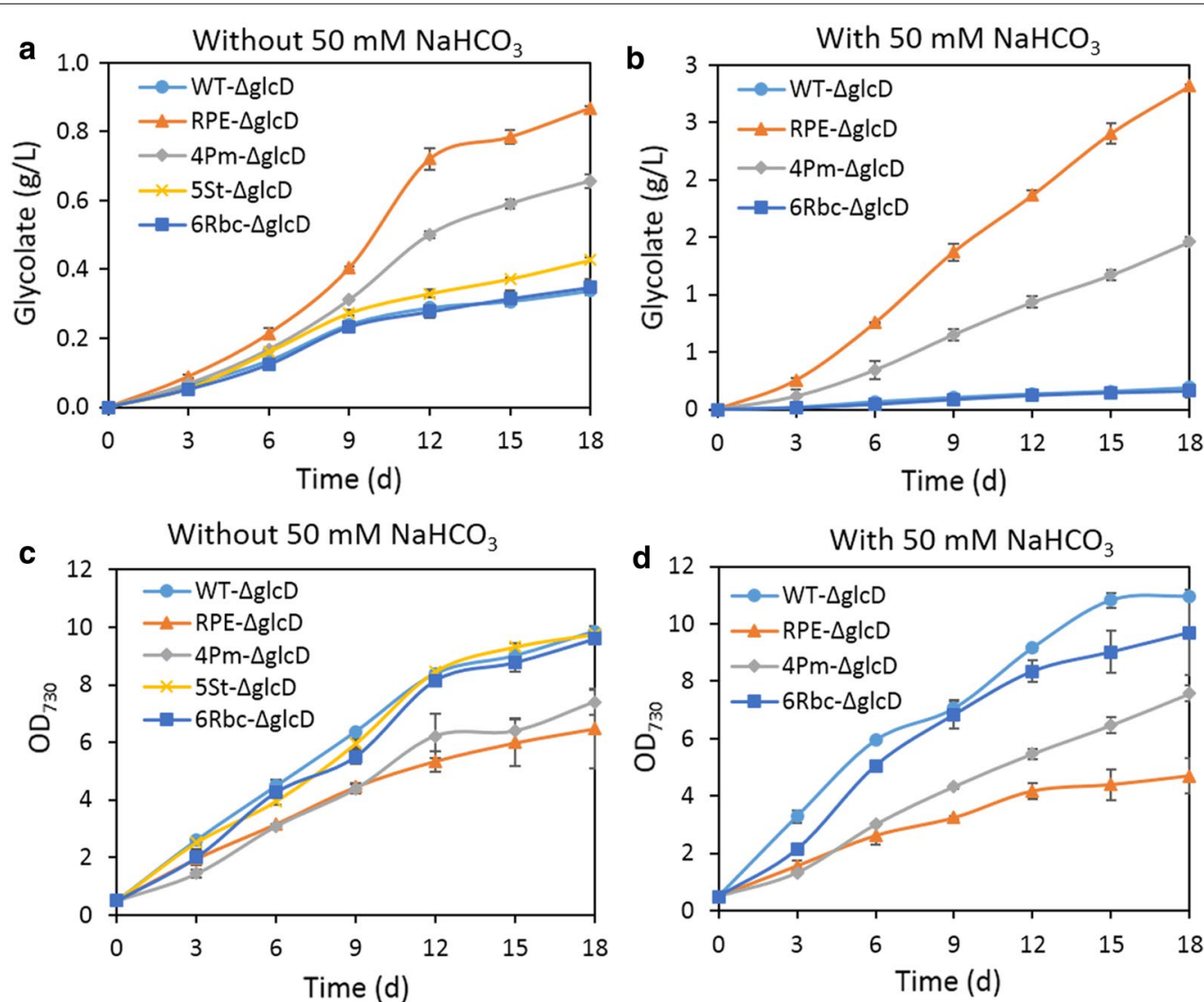

Fig. 3 Overexpression of Form II Rubisco increased glycolate production. Glycolate production (a and $\mathbf{b})$, growth curve (c and $\mathbf{d}$ ) of the Synechocystis strains expressing different Form II Rubiscos. The cells were cultivated without or with $50 \mathrm{mM} \mathrm{NaHCO}_{3}$ at $30{ }^{\circ} \mathrm{C}$ under $100 \mu \mathrm{mol}$ photons $\mathrm{m}^{-2} \mathrm{~s}^{-1}$ light intensity. Error bars represent standard deviations from biological triplicates conducted in three independent experiments 
this end, three Form II Rubiscos from Riftia pachyptila endosymbiont (RPE Rubisco), Phaeospirillum molischianum (4Pm Rubisco) and Sedimenticola thiotaurini (5St Rubisco) were selected and individually overexpressed by using the strong promoter $\mathrm{P}_{\mathrm{cpc560}}$ in strain WT- $\Delta$ glcD (Table 1 ), resulting in strains $\mathrm{RPE}-\Delta$ glcD, $4 \mathrm{Pm}-\Delta \mathrm{glcD}$ and $5 \mathrm{St}-\Delta \mathrm{glcD}$, respectively (Additional file 1: Fig. S1).

Subsequently, glycolate production of these three strains were determined without additional $\mathrm{NaHCO}_{3}$, which seemed to be more favorable for strain WT- $\Delta$ glcD to produce glycolate. After 18 days of cultivation, strain $5 \mathrm{St}-\Delta$ glcD produced $0.43 \mathrm{~g} / \mathrm{L}$ glycolate, which is not significantly higher than that of strain WT- $\Delta$ glcD (Fig. 3a). Moreover, no significant difference on growth was observed between them (Fig. 3c). This incapacity for increasing glycolate production could be attributed to the undetectable expression and assembly of 5St Rubisco (Additional file 1: Fig. S4a). In contrast, glycolate production were dramatically enhanced in strains RPE- $\Delta$ glcD and $4 \mathrm{Pm}-\Delta$ glcD (Fig. 3a). After 18 days of cultivation, strain $4 \mathrm{Pm}-\Delta$ glcD produced $0.66 \mathrm{~g} / \mathrm{L}$ of glycolate, about twofold of strain WT- $\Delta \mathrm{glcD}$, while strain RPE$\Delta$ glcD produced $0.87 \mathrm{~g} / \mathrm{L}$ of glycolate, 2.6 -fold of strain WT- $\Delta$ glcD (Fig. 3a). However, the growth of strains RPE- $\Delta \mathrm{glcD}$ and $4 \mathrm{Pm}-\Delta \mathrm{glcD}$ were significantly impaired (Fig. 3c). The expression and assembly of RPE Rubisco and $4 \mathrm{Pm}$ Rubisco were also detected (Additional file 1: Fig. S4a). RPE Rubisco was copiously overexpressed and well assembled. By contrast, 4Pm Rubisco was successfully overexpressed but not assembled well. This explained their different capacity on enhancement of glycolate production. Taken together, these results showed that overexpression of Form II Rubisco indeed increased glycolate production.

\section{Supply of $\mathrm{NaHCO}_{3}$ increased glycolate production by strains RPE- $\Delta$ glcD and $4 \mathrm{Pm}-\Delta$ glcD}

As mentioned above, glycolate production by strain WT- $\Delta$ glcD decreased when supplied with $50 \mathrm{mM}$ $\mathrm{NaHCO}_{3}$ (Fig. 2). Thus, we further investigated whether glycolate production of strains RPE- $\Delta$ glcD and $4 \mathrm{Pm}-\Delta$ glcD would also be repressed when supplied with $50 \mathrm{mM} \mathrm{NaHCO}_{3}$.

Surprisingly, glycolate production by strains RPE$\Delta \mathrm{glcD}$ and $4 \mathrm{Pm}-\Delta \mathrm{glcD}$ were not decreased, but instead sharply increased when $\mathrm{NaHCO}_{3}$ was available (Fig. 3b). Strain $4 \mathrm{Pm}-\Delta \mathrm{glcD}$ produced $1.46 \mathrm{~g} / \mathrm{L}$ of glycolate in 18 days when supplemented with $50 \mathrm{mM} \mathrm{NaHCO}_{3}$, which is about 7.7-fold of the titer of strain WT- $\Delta$ glcD under the same condition (Fig. $3 \mathrm{~b}$ ). This is also more than twofold of the titer produced by strain $4 \mathrm{Pm}-\Delta \mathrm{glcD}$ without additional $\mathrm{NaHCO}_{3}$. Additionally, 4Pm Rubisco assembled better upon the addition of $50 \mathrm{mM} \mathrm{NaHCO}$, which could contribute to the increased glycolate production of strain $4 \mathrm{Pm}-\Delta \mathrm{glcD}$ (Additional file 1: Fig. S4b). Among these three strains, strain RPE- $\Delta$ glcD was inarguably the best glycolate producer, generating $2.82 \mathrm{~g} / \mathrm{L}$ after 18 days of cultivation, about 15 -fold of the titer of strain WT- $\Delta$ glcD under the same growth condition (Fig. 3b). Moreover, the expression and assembly of RPE did not differ upon the addition of $\mathrm{NaHCO}_{3}$ (Additional file 1: Fig. S4b), suggesting that the increased glycolate production was not related to the assembly of RPE Rubisco. However, the growth of strains RPE- $\Delta$ glcD and $4 \mathrm{Pm}-\Delta \mathrm{glcD}$ were also significantly impaired under this condition (Fig. 3d).

Thus, we further investigated glycolate production of strain RPE- $\Delta$ glcD when supplied with different concentration of $\mathrm{NaHCO}_{3}$. Glycolate production of strain RPE- $\Delta$ glcD increased along with increasing the concentration of $\mathrm{NaHCO}_{3}$, and approached a plateau of $2.84 \mathrm{~g} / \mathrm{L}$ when supplied with $30 \mathrm{mM} \mathrm{NaHCO}$ (Fig. 4a). Notably, the growth of strain RPE- $\Delta$ glcD gradually reduced along with the increased glycolate production (Fig. 4b). The intracellular glycolate concentration in RPE- $\Delta$ glcD was also increased, from $5.6 \mu \mathrm{mol} \mathrm{L}^{-1} \mathrm{OD}_{730}{ }^{-1}$ in the absence of $\mathrm{NaHCO}_{3}$, to $10.4 \mu \mathrm{mol} \mathrm{L} \mathrm{L}^{-1} \mathrm{OD}_{730}{ }^{-1}$ when adding $50 \mathrm{mM} \mathrm{NaHCO}$ (Additional file 1: Fig. S2). It was previously reported that intracellular accumulation of glycolate is toxic to the cell (Eisenhut et al. 2008). The retarded growth of strain RPE- $\Delta$ glcD upon adding increased concentration of $\mathrm{NaHCO}_{3}$ was probably related to the elevated intracellular glycolate concentration.

\section{Supply of $\mathrm{CO}_{2}$ decreased glycolate production by strain RPE- $\Delta$ glcD}

Cyanobacteria can use both $\mathrm{HCO}_{3}{ }^{-}$and $\mathrm{CO}_{2}$ as external inorganic carbon source (Price et al. 2008, Price 2011). As supply of $\mathrm{HCO}_{3}{ }^{-}$increased glycolate production of strains RPE- $\Delta$ glcD and $4 \mathrm{Pm}-\Delta$ glcD, we then wondered what would be the effect if supplying $\mathrm{CO}_{2}$. Since strain RPE- $\Delta$ glcD produced much higher titer of glycolate than strain $4 \mathrm{Pm}-\Delta \mathrm{glcD}$, we chose strain RPE- $\Delta \mathrm{glcD}$ to study the effect of $\mathrm{CO}_{2}$.

To this end, the external organic carbon supplied was changed from $\mathrm{NaHCO}_{3}$ to $\mathrm{CO}_{2}$. The glycolate production and growth of strain RPE- $\Delta$ glcD were evaluated under $1 \%$ or $3 \% \mathrm{CO}_{2}$ (Fig. $4 \mathrm{c}$ and d). After 12 days of cultivation, strain RPE- $\Delta$ glcD produced $0.87 \mathrm{~g} / \mathrm{L}$ glycolate under $1 \%$ $\mathrm{CO}_{2}$, and the glycolate titer decreased to $0.47 \mathrm{~g} / \mathrm{L}$ under $3 \% \mathrm{CO}_{2}$ (Fig. 4c). Additionally, the growth of strain RPE$\Delta \mathrm{glcD}$ increased positively with increasing the $\mathrm{CO}_{2}$ level (Fig. 4d). The increased growth and reduced glycolate production of RPE- $\Delta$ glcD together indicated that supply of $\mathrm{CO}_{2}$ enhanced the carboxylation reaction of RPE and consequently inhibited the oxygenation reaction. 

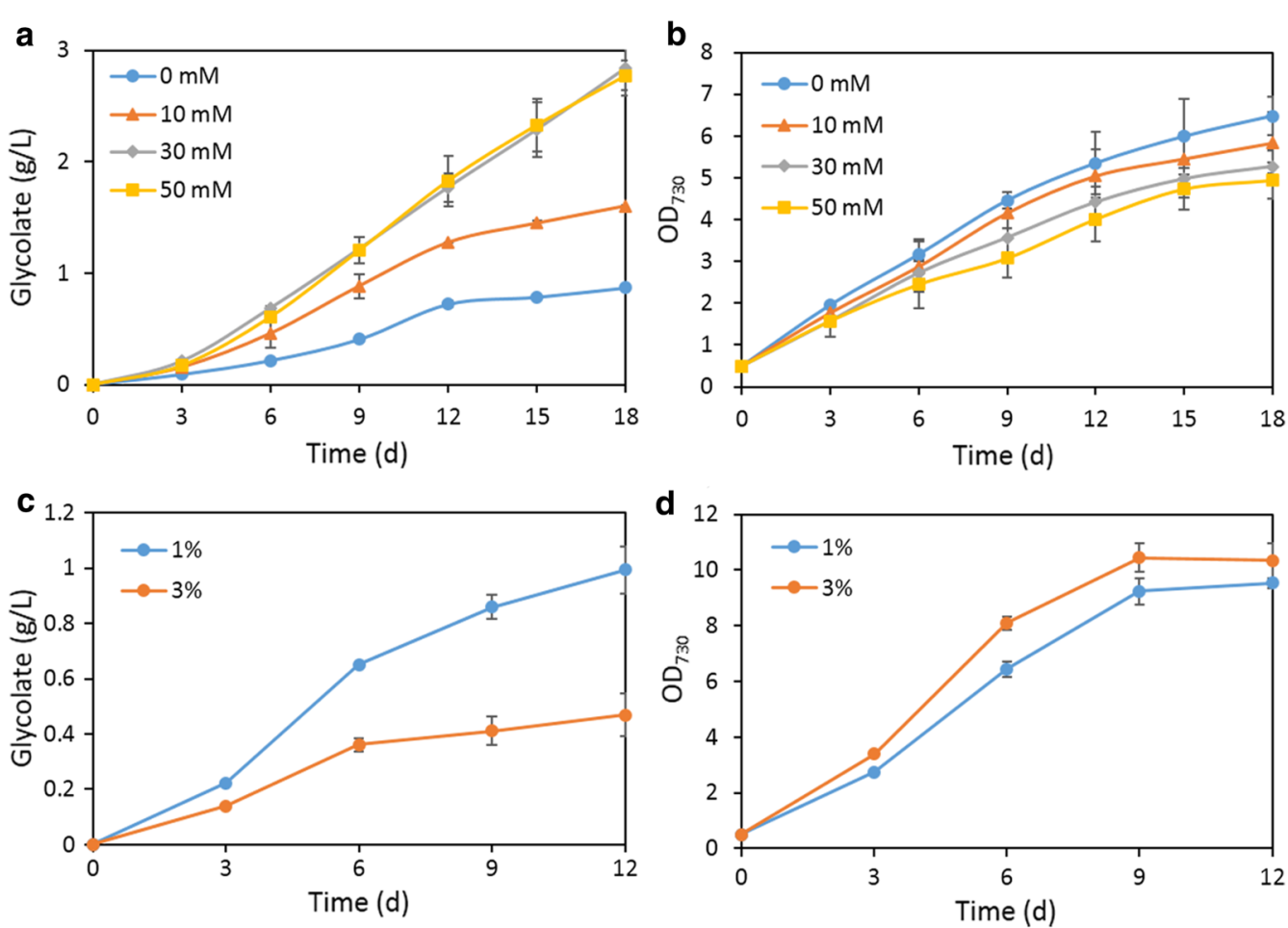

Fig. 4 Glycolate production and growth profile of strain RPE- $\triangle \mathrm{glcD}$ with supply of $\mathrm{NaHCO}_{3}$ or $\mathrm{CO}_{2}$. Cells were cultivated with different concentrations of $\mathrm{NaHCO}_{3}(\mathbf{a}$ and $\mathbf{b})$ or $\mathrm{CO}_{2}(\mathbf{c}$ and $\mathbf{d})$ at $30^{\circ} \mathrm{C}$ under $100 \mu \mathrm{mol}$ photons $\mathrm{m}^{-2} \mathrm{~s}^{-1}$ light intensity. Error bars represent standard deviations from biological triplicates conducted in three independent experiments

\section{RPE rubisco is located in the cytosol}

The enhanced glycolate production indicated the active oxygenation reaction catalyzed by RPE Rubisco and 4Pm Rubisco. This suggested that they are probably located in the cytosol rather than in the carboxysome as the $\mathrm{O}_{2}$ concentration in cytosol is much higher. To provide direct evidence, we visualized their locations in vivo by fluorescent labelling. We first tried to carry out the colocalization analysis by labelling RPE Rubisco with cyan fluorescent protein (CFP) and 6RBC with yellow florescent protein (YFP). RPE Rubisco was labelled with CFP at its $\mathrm{C}$-terminal (termed as RPE-CFP). YFP was fused to the C-terminal of the large subunit of $6 R B C$ (termed as 6RBCL-YFP). RPE-CFP and 6RBCL-YFP were individually expressed in the WT strain to give single fluorescent signal and co-expressed in the WT strain to test whether these two fluorescent signals could be overlayed together. However, the fluorescent signals of RPF-CFP and 6RBCLYFP were too week to give the location information (data not shown).

We next fused green fluorescent protein (GFP) to the C-terminal of RPE Rubisco or the large subunit of 6RBC Rubisco, termed as RPE-GFP and 6RBCL-GFP, respectively. RPE-GFP and 6RBCL-GFP were individually expressed in the WT strain to give single fluorescent signal. Meanwhile, the red fluorescence of endogenous chlorophyll-a of Synechocystis was used to indicate the shape of the whole cell (Cameron et al. 2013). RPE-GFP gave rise to a large single fluorescent punctum at the cell polar, suggesting that RPE proteins intended to aggregate at the edge of cell (Fig. 5a). By contrast, 6RBCL-GFP intended to exhibit several fluorescent spots at a more central position within the cell, indicating the locations of mature carboxysomes, which was in agreement with the previous report (Fig. 5b) (Cameron et al. 2013). The different positions of fluorescent signals between RPEGFP and 6RBCL-GFP indicated that RPE is not located in the carboxysome where 6RBCL-GFP resides. The bacterial Form II Rubisco from Rhodospirillum rubrum was previously expressed in the $\Delta$ rbc strain of Synechocystis (Durao et al. 2015). The resulting mutant could not support the biogenesis of carboxysome and photoautotrophic growth at ambient $\mathrm{CO}_{2}$ concentration (Durao et al. 2015). Thus, it is conceivable that the aggregate of RPE-GFP observed here is most likely in the cytosol.

\section{Discussion}

The oxygenase function of Rubisco and the ensuing photorespiration have long been regarded as one of the obstacles to improve the photosynthesis efficiency (South 
a

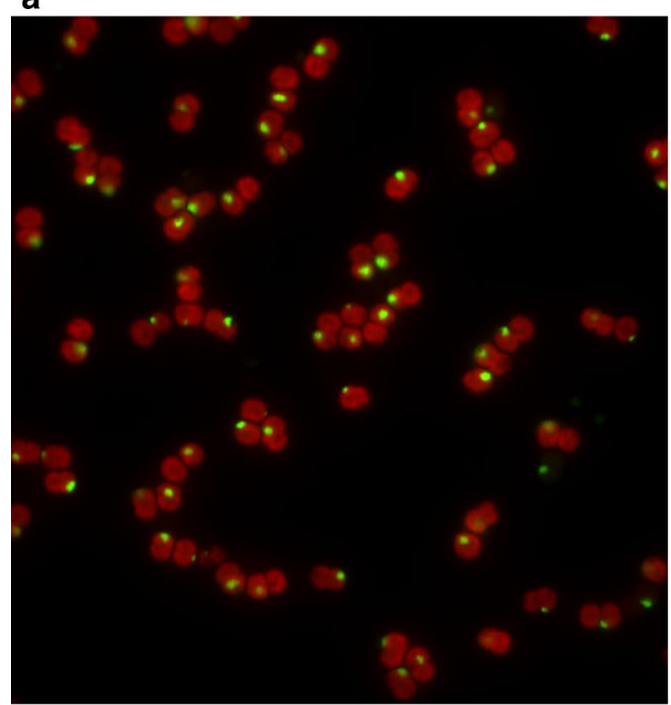

b

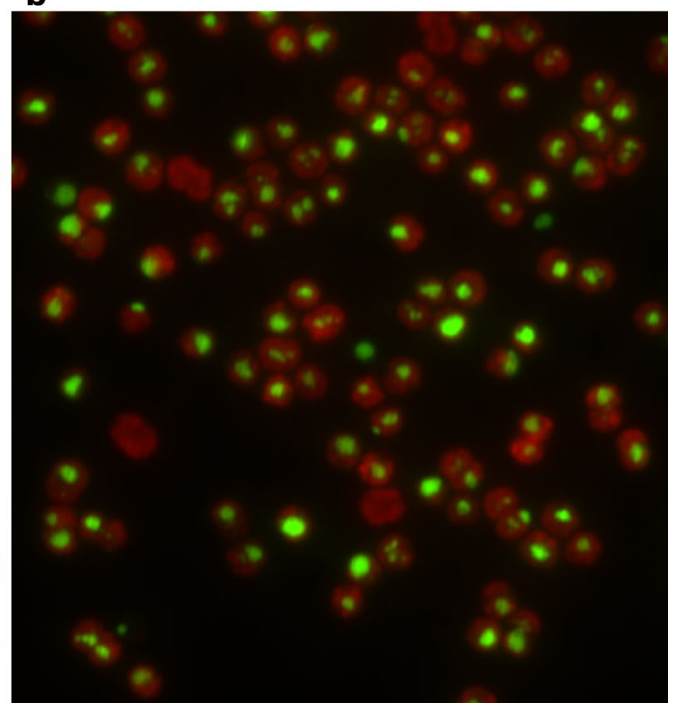

Fig. 5 Location of RPE Rubisco in the Synechocystis strain. The fluorescent signal of RPE-GFP (a) and 6RBCL-GFP (b). GFP was fused to the C-terminal of RPE Rubisco or the large subunit of 6RBC Rubisco. RPE-GFP and 6RBCL-GFP were individually expressed in the WT strain. The foci of 6RBC-GFP represents the location of mature carboxysomes. The red fluorescence of endogenous chlorophyll-a was used to indicate the shape of the whole cell

et al. 2018; Hu et al. 2019; Luan et al. 2020). Cumulative studies have attempted to inhibit even avoid the occurrence of the oxygenation reaction of Rubisco but gained limited progress (Erb and Zarzycki 2018, Davidi et al. 2020). Here, as the oxygenation product of Rubisco is involved in the overall carbon metabolism, we utilized the oxygenation activity of Form II Rubisco for production of glycolate, a versatile chemical with extensive industrial applications, from $\mathrm{CO}_{2}$ in Synechocystis.

In Synechocystis, glycolate can only be generated from 2PG, the direct product of the oxygenation reaction of Rubisco. Glycolate is then converted to glyoxylate and subsequently metabolized by three branched routes including the plant-like photorespiratory cycle, the bacterial glycerate pathway and the complete decarboxylation of glyoxylate to $\mathrm{CO}_{2}$ (Eisenhut et al. 2008a, b). In the first instance, glycolate production was primarily achieved by inactivation of two forms of glycolate dehydrogenases which are responsible for converting glycolate to glyoxylate. As glycolate metabolism is completely inactivated, the resulting strain WT- $\Delta$ glcD produced glycolate irrespective of the provision of $\mathrm{NaHCO}_{3}$. This indicated that Rubisco is performing the oxygenase function despite the active $\mathrm{CO}_{2}$-concentrating mechanism (CCM) and the abundance of inorganic carbon, which is also proved in the earlier studies (Eisenhut et al. 2006, Eisenhut et al. 2008a, b). It is still under discussion whether cytosolic Rubisco, which is in the various stages of assembly during carboxysome biogenesis, is responsible for this oxygenase activity, or whether significant amounts of $\mathrm{O}_{2}$ indeed enter the carboxysome (Espie and Kimber 2011, Burnap et al. 2015). Since overexpression of 6RBC Rubisco showed no effect on glycolate production, it is conceivable that the availability of $\mathrm{O}_{2}$ is limited in carboxysome.

Additionally, inactivation of glycolate metabolism was reported to render a high- $\mathrm{CO}_{2}$-requiring (HCR) phenotype which means the mutant was not able to grow at ambient $\mathrm{CO}_{2}$ level (Eisenhut et al. 2008a, b). This HCR phenotype was presumably ascribed to the intracellular accumulation of toxic amounts of glycolate (Eisenhut et al. 2008a, b). It was reported that the intracellular glycolate concentration in the mutant increased to a much higher level within a few hours after the mutant was transferred from $\mathrm{HC}\left(5 \% \mathrm{CO}_{2}\right)$ to $\mathrm{LC}$ (air, $0.035 \% \mathrm{CO}_{2}$ ) condition (Eisenhut et al. 2008a, b). Interestingly, strain WT- $\Delta$ glcD that we constructed did not exhibit the HCR phenotype (Additional file 1: Fig. S3). Further investigation suggested that strain WT- $\Delta$ glcD did accumulate intracellular glycolate, but more than $99 \%$ of glycolate was excreted to the culture (Fig. 2 and Additional file 1: Fig. S2). Glycolate excretion was previously observed in some filamentous cyanobacterial strains but not in Synechocystis, nor in mutant with HCR phenotype (Eisenhut et al. 2006, Eisenhut et al. 2008a, b). It is likely that glycolate excretion of strain WT- $\Delta$ glcD helped maintain the intracellular glycolate concentration at a lowel, which allows the cell to grow normally at ambient $\mathrm{CO}_{2}$ level, without displaying the HCR phenotype. It is worthy 
to further investigate the underlying mechanism of glycolate excretion of strain WT- $\Delta$ glcD.

To further increase glycolate production, we identified the rate-limiting step by measuring the intracellular 2 PG and glycolate concentrations of strain WT- $\Delta$ glcD. The result indicated that the conversion from 2PG to glycolate is fully active. As such, the oxygenase activity of Rubisco is the bottleneck of glycolate production, thus its activity needs to be increased. Accessibility to molecular oxygen is the prerequisite for the oxygenation reaction of Rubisco to occur. Overexpression of the native carboxysome-located 6RBC Rubisco of Synechocystis in strain WT- $\Delta$ glcD did not increase glycolate production, indicating that the oxygenation reaction of $6 \mathrm{RBC}$ Rubisco is hampered in the carboxysome which is a $\mathrm{CO}_{2}$-rich but $\mathrm{O}_{2}$-sheilding microcompartment (Price et al. 2008, Espie and Kimber 2011, Price 2011).

As compared to the carboxysome, the $\mathrm{CO}_{2}$ concentration in the cytosol is much lower. To provide the gradient for inward diffusion of $\mathrm{CO}_{2}$ and minimize its leakage from cell, cyanobacteria accumulates $\mathrm{HCO}_{3}{ }^{-}$but not $\mathrm{CO}_{2}$ in the cytosol and maintains a chemical equilibrium in favor of $\mathrm{HCO}_{3}{ }^{-}$over $\mathrm{CO}_{2}$ (Price et al. 2008, Price 2011, Burnap et al. 2015). Thus, the low- $\mathrm{CO}_{2}$-level cytosol might be a more favorable environment for the oxygenation reaction of Rubisco to occur. Additionally, as cyanobacteria performs oxygenic photosynthesis (Moroney et al. 2013), the photosynthetic evolved $\mathrm{O}_{2}$ from photosystem II located at the thylakoid membrane may also contribute to glycolate production. Overexpression of an exogenous Form II Rubisco located in the cytosol indeed increased glycolate production. Among the three forms of Rubisco, there are three reasons why we consider Form II Rubiscos promising candidates for glycolate production. First, the specificity of Form II Rubisco was reported to be extremely low, and thus can catalyze the oxygenation reaction more easily (Davidi et al. 2020). Second, Form II Rubisco is not packaged in the carboxysome, as they do not support the carboxysome biogenesis (Baker et al. 1998, Durao et al. 2015). Third, Form II Rubiscos are structurally simple, comprising only a large subunit and commonly forming an $\mathrm{L}_{2}$ or $\mathrm{L}_{6}$ oligomer (Davidi et al. 2020).

In this study, three Form II Rubiscos were selected and individually overexpressed in strain WT- $\Delta \mathrm{glcD}$. Among them, both RPE Rubisco and 4Pm Rubisco increased glycolate production irrespective of carbon supplement. Strain RPE- $\Delta$ glcD showed the highest glycolate titer of $2.8 \mathrm{~g} / \mathrm{L}$ after 18 days of cultivation when supplied with $50 \mathrm{mM} \mathrm{NaHCO}$ (Fig. 3b). Remarkably, it compares favorably over the majority of products synthesized from $\mathrm{CO}_{2}$ in cyanobacteria as listed in Table 2 (Titer $>1 \mathrm{~g} / \mathrm{L}$ ). A more detailed list of products can be found in reviews referenced (Oliver et al. 2014, Lai et al. 2015, Gao et al. 2016a, b; Knoot et al. 2018). This indicated that the deceptively wasteful and undesired oxygenase activity of Rubisco has immense yet undeveloped ability with regard to photosynthetic bioproduction application.

It is interesting that supply of $\mathrm{NaHCO}_{3}$ and $\mathrm{CO}_{2}$ exhibit different effects on glycolate production by strain RPE- $\Delta$ glcD, as $\mathrm{NaHCO}_{3}$ supply increased glycolate production while $\mathrm{CO}_{2}$ supply decreased glycolate production. This could be related to the different manners of $\mathrm{HCO}_{3}{ }^{-}$and $\mathrm{CO}_{2}$ entering the cell and the $\mathrm{CCM}$ applied by cyanobacteria. $\mathrm{HCO}_{3}{ }^{-}$is transported into the cytosol by the transporters located at the cytoplasmic membrane. The majority then enters the carboxysome and the sequestered carbonic anhydrase (CA) converts it to $\mathrm{CO}_{2}$. $\mathrm{RuBP}$ enters the carboxysome and reacts with $\mathrm{CO}_{2}$ catalyzed by the native Rubisco, generating two molecules of

Table 2 Chemicals synthesized from $\mathrm{CO}_{2}$ by engineered cyanobacteria, with titers higher than $1 \mathrm{~g} / \mathrm{L}$

\begin{tabular}{llll}
\hline Product & Strain & Titer & References \\
\hline Sucrose & Synechococcus elongatus UTEX 2973 & $8 \mathrm{~g} / \mathrm{L}$ & Lin et al. (2020) \\
Trehalose & Synechococcus elongatus PCC 7942 & $5.7 \mathrm{~g} / \mathrm{L}$ & Qiao et al. (2020) \\
Ethanol & Synechocystis sp. PCC 6803 & $5.5 \mathrm{~g} / \mathrm{L}$ & Gao et al. (2012) \\
1-Butanol & Synechocystis sp. PCC 6803 & $4.8 \mathrm{~g} / \mathrm{L}$ & Liu et al. (2019) \\
Glycolate & Synechocystis sp. PCC 6803 & $2.81 \mathrm{~g} / \mathrm{L}$ & This study \\
2,3-Butanediol & Synechococcus elongatus PCC 7942 & $2.38 \mathrm{~g} / \mathrm{L}$ & Oliver et al. (2013) \\
D-Lactic acid & Synechocystis sp. PCC 6803 & $2.17 \mathrm{~g} / \mathrm{L}$ & Varman et al. (2013) \\
Isoprene & Synechococcus elongatus PCC 7942 & $1.26 \mathrm{~g} / \mathrm{L}$ & Gao et al. (2016a, b) \\
1,3-Propanediol & Synechococcus elongatus PCC 7942 & $1.22 \mathrm{~g} / \mathrm{L}$ & Hirokawa et al. (2017) \\
(R)-3-Hydroxybutyrate & Synechocystis sp. PCC 6803 & $1.84 \mathrm{~g} / \mathrm{L}$ & Wang et al. (2018) \\
Isobutyraldehyde & Synechococcus elongatus PCC 7942 & $1.1 \mathrm{~g} / \mathrm{L}$ & Atsumi et al. (2009) \\
Mannitol & Synechococcus sp. PCC 7002 & $1.1 \mathrm{~g} / \mathrm{L}$ & Jacobsen et al. (2014) \\
\hline
\end{tabular}


3PGA. 3PGA escapes from the carboxysome and regenerates RuBP in the cytosol via CBB cycle (Fig. 6a). When supplied with $\mathrm{NaHCO}_{3}$, the increased $\mathrm{HCO}_{3}{ }^{-}$availability generally facilitates the carbon fixation of the native Rubisco in the carboxysome and results in the enhanced $\mathrm{RuBP}$ regeneration via $\mathrm{CBB}$ cycle (Fig. 6a). As regenerated in the cytosol, RuBP is preferentially oxygenated by $\mathrm{RPE}$. Accordingly, less RuBP is channeled to biomass production, and the growth of strain RPE- $\Delta \mathrm{glcD}$ is impaired upon supplementation of additional $\mathrm{NaHCO}_{3}$.

Furthermore, due to the absence of $\mathrm{CA}$ in the cytosol, the spontaneous conversion of $\mathrm{HCO}_{3}{ }^{-}$to $\mathrm{CO}_{2}$ in the cytosol is much slower than the diffusion of $\mathrm{CO}_{2}$ across the cytoplasmic membrane (Mangan et al. 2016). This means that supply of $\mathrm{NaHCO}_{3}$ could not sharply raise the
$\mathrm{CO}_{2}$ concentration in the cytosol. RPE Rubisco is identified from the chemolithoautotrophic symbiont in the trophosome of giant tubeworm $R$. pachyptila living at the deep-sea hydrothermal vents where the partial pressure of $\mathrm{CO}_{2}$ can reach up to $2.9 \mathrm{kPa}$ (Lutz et al. 1994). The internal $\mathrm{CO}_{2}$ concentration of $R$. pachyptila can approach up to $31 \mathrm{mM}$ relying on the high concentration of CA in the worm's plume and trophosome tissue (Childress et al. 1993). Therefore, it is possible that RPE Rubisco exhibits relatively low affinity to $\mathrm{CO}_{2}$. Thus, the oxygenase activity of RPE is not inhibited even when supplied with $50 \mathrm{mM}$ $\mathrm{NaHCO}_{3}$.

As an uncharged small molecule, $\mathrm{CO}_{2}$ can cross the cell membrane by diffusion (Price et al. 2008, Price 2011). Meanwhile, RPE Rubisco was not scattered inside the cell

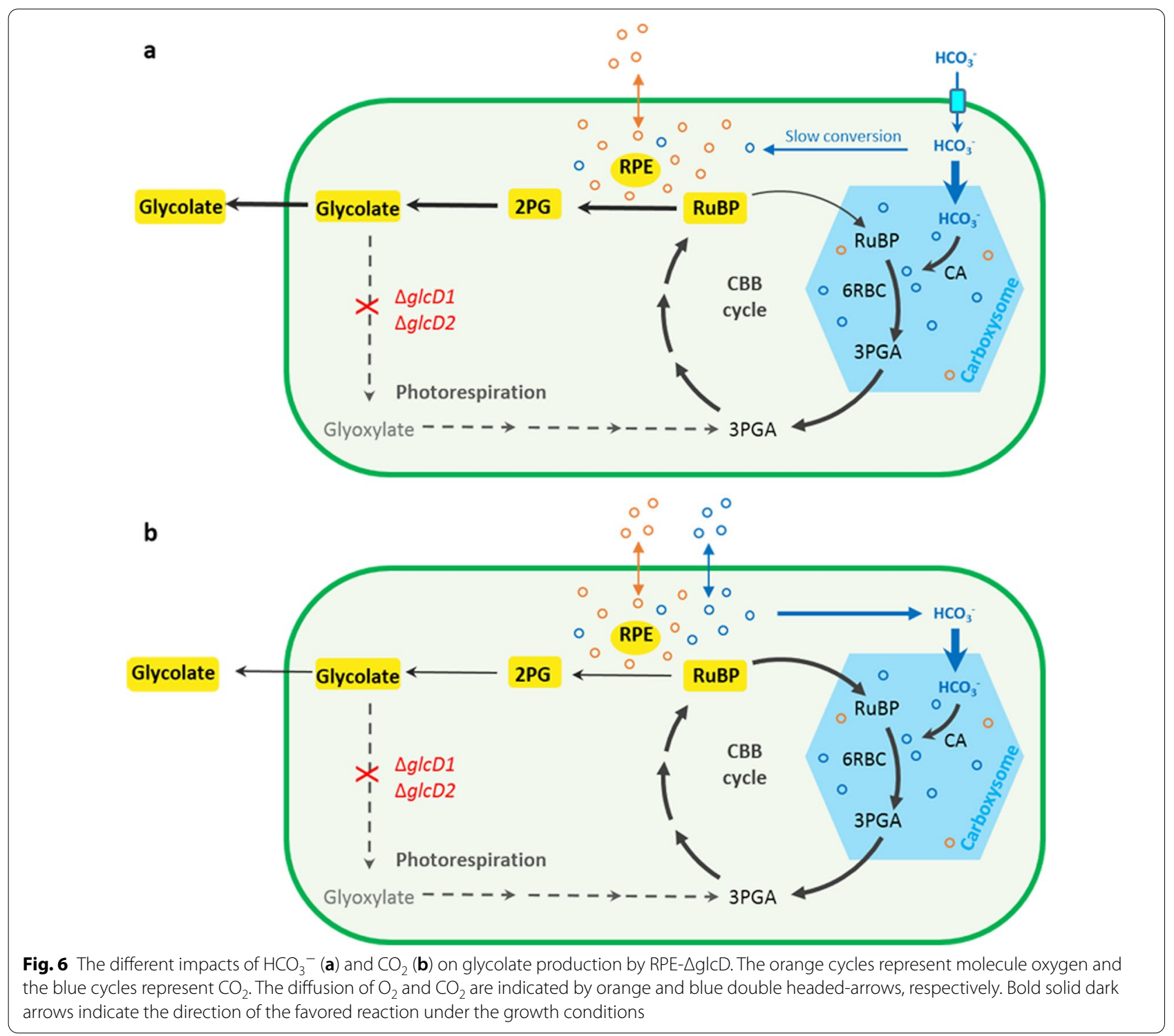


but aggregated near the cytoplasmic membrane. When supplied with $\mathrm{CO}_{2}$, the relative concentration of $\mathrm{CO}_{2}$ around RPE Rubisco is raised (as $\mathrm{O}_{2}$ concentration is not changed) (Fig. 6b). Thus, oxygenation is inhibited and carboxylation is enhanced alone with the increased availability of $\mathrm{CO}_{2}$. As a consequence, more $\mathrm{RuBP}$ is channeled to carbon fixation via $\mathrm{CBB}$ cycle, leading to increased cell growth and decreased glycolate production of strain RPE- $\Delta$ glcD when supplied with $\mathrm{CO}_{2}$ (Fig. 6b).

In summary, we demonstrated that the oxygenase function of Form II Rubisco could be explored for production of chemicals, e.g. glycolate, from $\mathrm{CO}_{2}$. Blocking the metabolism of photorespiration pathway led to glycolate production, and the efficiency for producing glycolate can be significantly improved when expressing Form II Rubisco in the cytosol. Thus, Form II Rubisco with distinct peculiarity can exert their versatile extraordinary capability in photosynthetic biosynthesis applications.

\section{Supplementary Information}

The online version contains supplementary material available at https://doi. org/10.1186/s13568-021-01224-6.

\section{Additional file 1.}

\section{Acknowledgements}

Not applicable.

\section{Authors' contributions}

$Y L$ and JZ designed the research. FY, JLZ, CZ performed the research. YL and FY wrote the manuscript. All authors read and approved the final manuscript.

\section{Funding}

This work was supported by Natural Science Foundation of China (31470231).

\section{Declarations}

Ethics approval and consent to participate

Not applicable.

\section{Consent for publication \\ Not applicable.}

\section{Competing interests}

The authors declare no conflicts of interest in regards to this manuscript.

\section{Author details}

'CAS Key Laboratory of Microbial Physiological and Metabolic Engineering, State Key Laboratory of Microbial Resources, Institute of Microbiology, Chinese Academy of Sciences, Beijing 100101, China. ${ }^{2}$ University of the Chinese Academy of Sciences, Beijing, China. ${ }^{3}$ CAS Key Laboratory of Microbial Physiological and Metabolic Engineering, State Key Laboratory of Transducer Technology, Institute of Microbiology, Chinese Academy of Sciences, Beijing 100101, China.

Received: 10 February 2021 Accepted: 26 April 2021

Published online: 08 May 2021

\section{References}

Alkim C, Trichez D, Cam Y, Spina L, François JM, Walther T (2016) The synthetic xylulose-1 phosphate pathway increases production of glycolic acid from xylose-rich sugar mixtures. Biotechnol Biofuels 9(1):201. https://doi.org/ 10.1186/s13068-016-0610-2

Atsumi S, Higashide W, Liao JC (2009) Direct photosynthetic recycling of carbon dioxide to isobutyraldehyde. Nat Biotechnol 27(12):1177-1180. https://doi.org/10.1038/nbt.1586

Baker SH, Jin S, Aldrich HC, Howard GT, Shively JM (1998) Insertion mutation of the Form I cbbl gene encoding ribulose bisphosphate carboxylase/ oxygenase (Rubisco) in Thiobacillus neapolitanus results in expression of Form II Rubisco, loss of carboxysomes, and an increased $\mathrm{CO}_{2}$ requirement for growth. J Bacteriol 180(16):4133-4139. https://doi.org/10.1128/JB.180. 16.4133-4139.1998

Burnap RL, Hagemann M, Kaplan A (2015) Regulation of $\mathrm{CO}_{2}$ concentrating mechanism in cyanobacteria. Life 5(1):348-371. https://doi.org/10.3390/ life5010348

Cameron J, Wilson S, Bernstein S, Kerfeld C (2013) Biogenesis of a bacterial organelle: the carboxysome assembly pathway. Cell 155(5):1131-1140. https://doi.org/10.1016/j.cell.2013.10.044

Childress JJ, Lee RW, Sanders NK, Felbeck H, Oros DR, Toulmond A, Desbruyeres D, Kennicutt MC, Brooks JJN (1993) Inorganic carbon uptake in hydrothermal vent tubeworms facilitated by high environmental $\mathrm{pCO}_{2}$. Nature 362(6416):147-149. https://doi.org/10.1038/362147a0

Davidi D, Shamshoum M, Guo Z, Bar-On YM, Prywes N, Oz A, Jablonska J, Flamholz A, Wernick DG, Antonovsky N, de Pins B, Shachar L, Hochhauser D, Peleg Y, Albeck S, Sharon I, Mueller-Cajar O, Milo R (2020) Highly active rubiscos discovered by systematic interrogation of natural sequence diversity. EMBO J 39(18):e104081. https://doi.org/10.15252/embj.20191 04081

Deng Y, Ma N, Zhu KJ, Mao Y, Wei XY, Zhao YY (2018) Balancing the carbon flux distributions between the TCA cycle and glyoxylate shunt to produce glycolate at high yield and titer in Escherichia coli. Metab Eng 46:28-34. https://doi.org/10.1016/j.ymben.2018.02.008

Deng Y, Mao Y, Zhang XJ (2015) Metabolic engineering of E. coli for efficient production of glycolic acid from glucose. Biochem Eng J 103:256-262. https://doi.org/10.1016/j.bej.2015.08.008

Durao P, Aigner H, Nagy P, Mueller-Cajar O, Hartl FU, Hayer-Hartl M (2015) Opposing effects of folding and assembly chaperones on evolvability of Rubisco. Nat Chem Biol 11(2):148-155. https://doi.org/10.1038/nchem bio. 1715

Eisenhut M, Huege J, Schwarz D, Bauwe H, Kopka J, Hagemann M (2008a) Metabolome phenotyping of inorganic carbon limitation in cells of the wild type and photorespiratory mutants of the cyanobacterium Synechocystis sp. strain PCC 6803. Plant Physiol 148(4):2109-2120. https://doi.org/ 10.1104/pp.108.129403

Eisenhut M, Kahlon S, Hasse D, Ewald R, Lieman-Hurwitz J, Ogawa T, Ruth W, Bauwe H, Kaplan A, Hagemann M (2006) The plant-like C2 glycolate cycle and the bacterial-like glycerate pathway cooperate in phosphoglycolate metabolism in cyanobacteria. Plant Physiol 142(1):333-342. https://doi. org/10.1104/pp.106.082982

Eisenhut M, Ruth W, Haimovich M, Bauwe H, Kaplan A, Hagemann M (2008b) The photorespiratory glycolate metabolism is essential for cyanobacteria and might have been conveyed endosymbiontically to plants. Proc Natl Acad Sci U S A 105(44):17199-17204. https://doi.org/10.1073/pnas.08070 43105

Erb TJ, Zarzycki J (2018) A short history of Rubisco: the rise and fall (?) of nature's predominant $\mathrm{CO}_{2}$ fixing enzyme. Curr Opin Biotechnol 49:100107. https://doi.org/10.1016/j.copbio.2017.07.017

Espie GS, Kimber MS (2011) Carboxysomes: cyanobacterial Rubisco comes in small packages. Photosynth Res 109(1):7-20. https://doi.org/10.1007/ s11120-011-9656-y

Fernie AR, Bauwe H (2020) Wasteful, essential, evolutionary stepping stone? The multiple personalities of the photorespiratory pathway. Plant J 102(4):666-677. https://doi.org/10.1111/tpj.14669

Gao X, Gao F, Liu D, Zhang H, Nie XQ, Yang C (2016a) Engineering the methylerythritol phosphate pathway in cyanobacteria for photosynthetic isoprene production from CO2. Energy Environ Sci 9(4):1400-1411. https:// doi.org/10.1039/C5EEO3102H

Gao XY, Sun T, Pei GS, Chen L, Zhang WW (2016b) Cyanobacterial chassis engineering for enhancing production of biofuels and chemicals. 
Appl Microbiol Biotechnol 100(8):3401-3413. https://doi.org/10.1007/ s00253-016-7374-2

Gao ZX, Zhao H, Li ZM, Tan XM, Lu XF (2012) Photosynthetic production of ethanol from carbon dioxide in genetically engineered cyanobacteria. Energy Environ Sci 5(12):9857-9865. https://doi.org/10.1039/C2EE22675H

Hirokawa Y, Maki Y, Hanai T (2017) Improvement of 1,3-propanediol production using an engineered cyanobacterium, Synechococcus elongatus by optimization of the gene expression level of a synthetic metabolic pathway and production conditions. Metab Eng 39:192-199. https://doi. org/10.1016/j.ymben.2016.12.001

Hu GP, Li Y, Ye C, Liu LM, Chen XL (2019) Engineering microorganisms for enhanced $\mathrm{CO}_{2}$ sequestration. Trends Biotechnol 37(5):532-547. https:// doi.org/10.1016/j.tibtech.2018.10.008

Jacobsen JH, Frigaard N-U (2014) Engineering of photosynthetic mannitol biosynthesis from $\mathrm{CO}_{2}$ in a cyanobacterium. Metab Eng 21:60-70. https:// doi.org/10.1016/j.ymben.2013.11.004

Jensen RG (2000) Activation of Rubisco regulates photosynthesis at high temperature and $\mathrm{CO}_{2}$. Proc Natl Acad Sci U S A 97(24):12937-12938. https:// doi.org/10.1073/pnas.97.24.12937

Knoot CJ, Ungerer J, Wangikar PP, Pakrasi HB (2018) Cyanobacteria: promising biocatalysts for sustainable chemical production. J Biol Chem 293(14):5044-5052. https://doi.org/10.1074/jbc.R117.815886

Koivistoinen OM, Kuivanen J, Barth D, Turkia H, Pitkänen J-P, Penttilä M, Richard P (2013) Glycolic acid production in the engineered yeasts Saccharomyces cerevisiae and Kluyveromyces lactis. Microb Cell Fact 12(1):82. https:// doi.org/10.1186/1475-2859-12-82

Lai MC, Lan El (2015) Advances in metabolic engineering of cyanobacteria for photosynthetic biochemical production. Metabolites 5(4):636-658 https://doi.org/10.3390/metabo5040636

Lin PC, Zhang FZ, Pakrasi HB (2020) Enhanced production of sucrose in the fast-growing cyanobacterium Synechococcus elongatus UTEX 2973. Sci Rep 10(1):390. https://doi.org/10.1038/s41598-019-57319-5

Lindberg P, Park S, Melis A (2010) Engineering a platform for photosynthetic isoprene production in cyanobacteria, using Synechocystis as the model organism. Metab Eng 12(1):70-79. https://doi.org/10.1016/j.ymben.2009. 10.001

Liu M, Ding YM, Xian M, Zhao G (2018) Metabolic engineering of a xylose pathway for biotechnological production of glycolate in Escherichia coli. Microb Cell Fact 17(1):51. https://doi.org/10.1186/s12934-018-0900-4

Liu XF, Miao R, Lindberg P, Lindblad P (2019) Modular engineering for efficient photosynthetic biosynthesis of 1-butanol from $\mathrm{CO}_{2}$ in cyanobacteria. Energy Environ Sci 12(9):2765-2777. https://doi.org/10.1039/C9EE01214A

Luan GD, Zhang SS, Lu XF (2020) Engineering cyanobacteria chassis cells toward more efficient photosynthesis. Curr Opin Biotechnol 62:1-6. https://doi.org/10.1016/j.copbio.2019.07.004

Lutz RA, Shank TM, Fornari DJ, Haymon RM, Lilley MD, Von Damm KL, Desbruyeres D (1994) Rapid growth at deep-sea vents. Nature 371(6499):663-664. https://doi.org/10.1038/371663a0

Mangan NM, Flamholz A, Hood RD, Milo R, Savage DF (2016) pH determines the energetic efficiency of the cyanobacterial $\mathrm{CO}_{2}$ concentrating mechanism. Proc Natl Acad Sci U S A 113(36):E5354-E5362. https://doi.org/10. 1073/pnas. 1525145113

Moroney JV, Jungnick N, DiMario RJ, Longstreth DJ (2013) Photorespiration and carbon concentrating mechanisms: two adaptations to high $\mathrm{O}_{2}$, low
$\mathrm{CO}_{2}$ conditions. Photosynth Res 117(1):121-131. https://doi.org/10.1007/ s11120-013-9865-7

Oliver JWK, Atsumi S (2014) Metabolic design for cyanobacterial chemical synthesis. Photosynth Res 120(3):249-261. https://doi.org/10.1007/ s11120-014-9997-4

Oliver JWK, Machado IMP, Yoneda H, Atsumi S (2013) Cyanobacterial conversion of carbon dioxide to 2,3-butanediol. Proc Natl Acad Sci U S A 110(4):1249. https://doi.org/10.1073/pnas.1213024110

Price GD (2011) Inorganic carbon transporters of the cyanobacterial $\mathrm{CO}_{2}$ concentrating mechanism. Photosynth Res 109(1-3):47-57. https://doi. org/10.1007/s11120-010-9608-y

Price GD, Badger MR, Woodger FJ, Long BM (2008) Advances in understanding the cyanobacterial $\mathrm{CO}_{2}$-concentrating-mechanism (CCM): functional components, Ci transporters, diversity, genetic regulation and prospects for engineering into plants. J Exp Bot 59(7):1441-1461. https://doi.org/10. 1093/jxb/erm112

Qiao Y, Wang WH, Lu XF (2020) Engineering cyanobacteria as cell factories for direct trehalose production from $\mathrm{CO}_{2}$. Metab Eng 62:161-171. https://doi. org/10.1016/j.ymben.2020.08.014

Rai S, Lucius S, Kern R, Bauwe H, Kaplan A, Kopka J, Hagemann M (2018) The Synechocystis sp. PCC 6803 genome encodes up to four 2-phosphoglycolate phosphatases. Front Plant Sci 9:1718. https://doi.org/10.3389/fpls. 2018.01718

Salusjärvi L, Toivari M, Vehkomäki M-L, Koivistoinen O, Mojzita D, Niemelä K, Penttilä M, Ruohonen L (2017) Production of ethylene glycol or glycolic acid from D-xylose in Saccharomyces cerevisiae. Appl Microbiol Biotechnol 101(22):8151-8163. https://doi.org/10.1007/s00253-017-8547-3

South PF, Cavanagh AP, Lopez-Calcagno PE, Raines CA, Ort DR (2018) Optimizing photorespiration for improved crop productivity. J Integr Plant Biol 60(12):1217-1230. https://doi.org/10.1111/jipb.12709

Spreitzer RJ, Salvucci ME (2002) Rubisco: structure, regulatory interactions, and possibilities for a better enzyme. Annu Rev Plant Biol 53(1):449-475. https://doi.org/10.1146/annurev.arplant.53.100301.135233

Varman AM, Yu Y, You L, Tang YJ (2013) Photoautotrophic production of D-lactic acid in an engineered cyanobacterium. Microb Cell Fact 12(1):117. https://doi.org/10.1186/1475-2859-12-117

Wang B, Xiong W, Yu J, Maness P-C, Meldrum DR (2018) Unlocking the photobiological conversion of $\mathrm{CO}_{2}$ to (R)-3-hydroxybutyrate in cyanobacteria. Green Chem 20(16):3772-3782. https://doi.org/10.1039/C8GC01208C

Zahoor A, Otten A, Wendisch VF (2014) Metabolic engineering of Corynebacterium glutamicum for glycolate production. J Biotechnol 192:366-375. https://doi.org/10.1016/j.jbiotec.2013.12.020

Zhan T, Chen Q, Zhang C, Bi CH, Zhang XL (2020) Constructing a novel biosynthetic pathway for the production of glycolate from glycerol in Escherichia coli. ACS Synth Biol 9(9):2600-2609. https://doi.org/10.1021/ acssynbio.0c00404

\section{Publisher's note}

Springer Nature remains neutral with regard to jurisdictional claims in published maps and institutional affiliations.

\section{Submit your manuscript to a SpringerOpen ${ }^{\odot}$ journal and benefit from:}

- Convenient online submission

- Rigorous peer review

- Open access: articles freely available online

- High visibility within the field

- Retaining the copyright to your article

Submit your next manuscript at $\boldsymbol{\nabla}$ springeropen.com 